\title{
A novel transcript of oil palm (Elaeis guineensis Jacq.), Eg707, is specifically upregulated in tissues related to totipotency
}

\begin{abstract}
In this study, we report the molecular characterization of clone Eg707 isolated from cell suspension culture of the oil palm. The deduced polypeptide of clone Eg707 is highly similar to an unknown protein from Arabidopsis thaliana. The presence of an Ald-Xan-dh-C2 superfamily domain in the deduced protein sequence suggested that Eg707 protein might be involved in abscisic acid biosynthesis. Eg707 might be present as a single copy gene in the oil palm genome. This gene is highly expressed in tissue cultured materials compared to vegetative and reproductive tissues, suggesting a role of this gene during oil palm somatic embryogenesis or at the early stages of embryo development. Expression analysis of Eg707 by RNA in situ hybridization showed that Eg707 transcripts were present throughout somatic embryo development starting from proembryo formation at the embryogenic callus stages till the maturing embryo stages. Since proembryo formation within the embryogenic callus is one of the first key factors in oil palm somatic embryo development, it is suggested that Eg707 could be used as a reliable molecular marker for detecting early stage of oil palm somatic embryogenesis.
\end{abstract}

Keyword: Oil palm; In situ hybridization; Histology; Totipotency; Embryogenic competence; Embryo development; Somatic embryogenesis; Abscisic acid 